\title{
Jeannette Littlemore, Metonymy: Hidden Shortcuts in Language, Thought and Communication, Cambridge
}

\author{
Lihua Zhu ${ }^{1}$, Zeqing WU ${ }^{2}$ \\ ${ }^{I}$ Faculty of Foreign Languages, Hunan Commerce University, Changsha, Hunan, China \\ ${ }^{1}$ Faculty of Modern and Medieval Languages, University of Cambridge, Cambridge, UK \\ ${ }^{2}$ Minzu University of China, Beijing, China
}

*Corresponding Author: Zeqing WU, Minzu University of China, Beijing, China

Metonymy has now been widely recognized and portrayed as a fundamental scheme of thought, which gets manifested at many levels of human experience, ranging from culture to culture, through everyday thinking and language. The book, Metonymy: hidden shortcuts in language, thought and communication, written by Jeannette Littlemore, mainly presents a full discussion of the different types of metonymy that have been identified in the literature, the different functions that metonymy performs, the contribution that it makes to successful communication in language and other forms of expression, the role that it plays in intercultural communication, and the types of misinterpretations that can occur in these contexts.

This book shows how metonymy operates, not just in language, but also in gesture, sign language, art, music, film and advertising. It explores the interactive role of metonymy in cross-cultural communication, along with the challenges it presents to language learners and translators.

Chapter 1 looks at what metonymy is, how it works and what it does.

Chapter 2 reveals a foundation to the book by outlining the taxonomies of key metonymy- producing relationships that have been identified in the cognitive linguistic literature. Key findings in this chapter are that the pragmatic functions of metonymy, which both context and linguistic form play key roles in determining the meanings of metonymic expressions

Chapter 3 explores a number of models as a basis for the analysis of metonymy as a dynamic phenomenon and attempts to classify metonymy to take a 'radial category' approach to the categorization of metonymy.

Chapters 4 focus on the functions of metonymy. It looks at some of the well-attested functions of metonymy, mainly in the linguistics literature including its referential functions and illocutionary functions.

Chapter 5 provides focuses on the less widely explored, creative, evaluative and attitudinal functions of metonymy in euphemism, dysphemism and hyperbole, irony and vagueness, and looks at how metonymy is used to convey evaluation, ideology and positioning. This chapter also extends the discussion to other forms of expression and communication, such as art, music, film and advertising to show different functions of metonymy in these different forms of expression.

Chapter 6 argues the difficulties involved in identifying metonymy in both language and other forms of expression. The difficulties involves in metonymy identification and notions such as 'contiguity' and 'ICMs' which are difficult to pin down. It also makes an attempt to automatically identify metonymy in language corpora.

In Chapter 7, the focus is on metonymy in the mind, with a meta-analysis of studies that have been made of metonymy comprehension and production in children and adults, neurological and psychological nature of metonymic processing and closes with a number of proposals for ways in which a more explicit focus on metonymic thinking might usefully be incorporated into psychological counseling. 
Jeannette Littlemore, Metonymy: Hidden Shortcuts in Language, Thought and Communication, Cambridge: Cambridge University Press, 2015.Pp. 370.Pb \$39.95

Chapter 8 discusses cross-linguistic and cross-cultural variation in metonymy and the challenges that this presents to language learners, how learners sometimes 'under-interpret' metonymy, seeing it as literal language, or 'over interpret' it, seeing it as metaphor. It looks at the impact of first language influence on metonymy production and considers the ways in which metonymy might be taught most effectively to learners of different languages. In this chapter, it presents challenges that metonymy to translators and ends with a discussion of the idea that metonymic thinking constitutes a translation strategy in its own right.

Chapter 9 provides a conclusion to the book, summing up the main characteristics of metonymy which include (1) metonymy is found in many other forms of expression besides language; (2) the metonymy serves a very wide range of rhetorical function; (3) metonymy is often used playfully and creatively and for humorous effect; (4) metonymy can be found in many different parts of speech; (5) metonymy is subtle, flexible, dynamic and contextually determined. The chapter closes with a forward-looking perspective, outlining ways in which metonymy can be used in potential real-world applications in the areas of psychotherapy, education, advertising, inter-cultural communication (in its broadest sense), second language teaching and translator training.

This book aims to present a full discussion of the different types of metonymy, the different functions that metonymy performs, the contribution that metonymy makes to successful communication in language and other forms of expression, and the types of misinterpretations that can occur with a range of socio-economic, political, cultural and linguistic features works alongside the cognitive principles. This book is accessibly and remarkably noteworthy for its meticulous juxta position of works from established metonymy scholars, some of whom have written briefly on the topic of metonymy or done work clearly relevant to both how and why people sometimes combine metonymy in their use of language, gesture, and multimodal media. The book, covering such a wide range of current trends and interests in metonymy studies, with its sensitivity to the nuances and contextual diversity of use, can be applauded as an applicable forerunner of Cognitive Linguistics.

Initially, the book has provided us with a comprehensive examination of metonymy and with various implications under the circumstance of little attention being given to the fact that metonymy is quite common. This book may bring the topic of metonymy center stage within the world of metonymy studies as ideal reflections of people's typical experiences in language, thought, and communication, rather than diametrically being a deviation from proper literal use, and thus the study of metonymy may offer significant insights into contrasting or even contradictory theories of metonymy. Secondly, several chapters in this book have outlined theoretical frameworks that may explain some of the various ways that metonymy is mixed in language and other forms of expression as instantiations. Thirdly, this book convincingly provides us with novel and broad perspectives on metonymy research in the discourse and quantitative corpus-based elucidation with a theoretical and practical account of its role in language and other forms of communication. Metonymy research at divergent contexts has not accumulated a complete theoretical system, as such, it is a new field which is worth exploring and discussing.

As opposed to those remarkable achievements, our top complaint about the book is that, being a multiple perspective about metonymy and with dispersed structure, this book fails to provide a systematic reproducible operational definition and introduction of metonymy and analyze the value and specific methods of research, which may result in beginners' comprehension difficulties. Even if the book has set the stage and sketched a roadmap for future research, its explanations appear much too misty, in particular in terms of psycho linguistic and neuro linguistic studies of metonymy. Not surprisingly, the discussion is only a rough sketch, but they do foreshadow the initiate promising avenues for further mature theorizing implementations and tentative applications. All existing theoretical schools should be reconciled into a unified and optimized model. Provided that the validity of any new theory or model requires that it is in variously used to describe and analyze a substantial number of cases, the working principles as well as their applicability need to be demonstrated in widely distinctive rather than monolithic fields, little has been conducted to contribute to deepening insights into underlying patterns of metonymy. Additionally, metonymy ought to be delved into in relation to their specific contexts, which are awaiting further demonstration and exploration. The next decade calls for theorists and practitioners to establish applicable descriptive models. It should filter 
Jeannette Littlemore, Metonymy: Hidden Shortcuts in Language, Thought and Communication, Cambridge: Cambridge University Press, 2015.Pp. 370.Pb \$39.95

out other plausible but inappropriate alternatives based upon a wide artillery of empirical experiments, which call upon empirical psychological evidence.

Overall, we found the engaging and thought-provoking book has provided a plethora of fertile starting points for future research in this emergent interdisciplinary area of metonymy studies. It has remarkably input food for thought to prompt and facilitate new tasks and discussions on metonymy.

Citation: Zeqing WU. "Jeannette Littlemore, Metonymy: Hidden Shortcuts in Language, Thought and Communication, Cambridge" International Journal on Studies in English Language and Literature (IJSELL), vol 7, no. 2, 2019, pp. 37-39. doi: http://dx.doi.org/10.20431/2347 -3134.0702005.

Copyright: (C) 2019 Authors. This is an open-access article distributed under the terms of the Creative Commons Attribution License, which permits unrestricted use, distribution, and reproduction in any medium, provided the original author and source are credited. 